\title{
Association between the NQO1 C609T Polymorphism with Hepatocellular Carcinoma Risk in the Chinese Population
}

\author{
Hong Zhao, Li-Wei Zou, Sui-Sheng Zheng*, Xiao-Ping Geng*
}

\begin{abstract}
Background: Associations between the NQO1 C609T polymorphism and hepatocellular carcinoma (HCC) risk are a subject of debate. We therefore performed the present meta-analysis to evaluate links with HCC susceptibility. Materials and Methods: Several major databases (PubMed, EBSCO), the Chinese national knowledge infrastructure (CNKI) and the Wanfang database were searched for eligible studies. Crude odds ratios (ORs) with $95 \%$ confidence intervals (CIs) were used to measure the strength of associations. Results: A total of 4 studies including 1,325 patients and 1,367 controls were identified. There was a significant association between NQO1 C609T polymorphism and HCC for all genetic models (allelic model: $O R=1.45,95 \% \mathrm{CI}=1.23-1.72, p<0.01$; additive model: $\mathrm{OR}=1.96,95 \% \mathrm{CI}=1.57-2.43, p<0.01$; dominant model: $\mathrm{OR}=1.62,95 \% \mathrm{CI}=1.38-1.91, p<0.01$; and recessive model: $\mathrm{OR}=1.53,95 \% \mathrm{CI}=1.26-1.84, p<0.01)$. On subgroup analysis, similarly results were identified in Asians. For Asians, the combined ORs and 95\% CIs were (allelic model: $O R=1.50,95 \% C I=1.24-1.82, p<0.01$; additive model: $\mathrm{OR}=\mathbf{2 . 1 1}, 95 \% \mathrm{CI}=1.48-3.01, p<0.01$; dominant model: $\mathrm{OR}=1.69,95 \% \mathrm{CI}=1.42-2.02, p<0.01$; and recessive model: $\mathrm{OR}=1.59,95 \% \mathrm{CI}=1.16-2.19, p<0.01)$. Conclusions: The current meta-analysis suggested that the NQO1 C609T polymorphism could be a risk factor for developing HCC, particularly in the Chinese population.
\end{abstract}

Keywords: Hepatocellular carcinoma - NQO1 - polymorphism - meta-analysis - Chinese

Asian Pac J Cancer Prev, 16 (5), 1821-1825

\section{Introduction}

Hepatocellular carcinoma (HCC) is the fifth most common cause of cancer and the third leading cause of cancer-related death worldwide (El-Serag and Rudolph, 2007; de Lope et al., 2012). Nearly 700,000 new cases of HCC are diagnosed each year and 600,000 deaths are recorded on the world (Farazi and DePinho, 2006). More than $75 \%$ of these cases occur in the Asia-Pacific region and China alone accounts for $55 \%$ cases of HCC worldwide (Parkin, 2001; Yuen et al., 2009).

It is mostly important to identify risk factors for $\mathrm{HCC}$, and aim at early develop interventions and promote our understanding of the complicated pathologic mechanisms of this disease. Chronic hepatitis B virus (HBV) and hepatitis $\mathrm{C}$ virus (HCV) infection is common environmental factors cause HCC. Moreover, other environmental risk factors including alcohol intake, cigarette smoking, and exposure to aflatoxin B1 are also the risk factor for HCC. However, only a fraction of HBsAg carriers eventually develop HCC and only $2.5 \%$ of HCV infected individuals develop HCC later in life (Bowen and Walker, 2005). Thus, host genetic factors may affect the development of HCC. Among the genetic factors, some single nucleotide polymorphisms (SNPs) have been regarded as HCC risk factors (Kato et al., 2005; Kim and Lee, 2005; Liu et al., 2013).

The cytosolic enzyme NAD(P)H: quinone oxidoreductase detoxification of environmental carcinogens(Dinkova-Kostova and Talalay, 2010; Siegel et al., 2012). It can catalyze the two-electron reduction of quinoid compounds and prevent the production of semiquinone free radicals and reactive oxygen species, thus protesting cells from oxidative damage(Siegel et al., 2012). NQO1 polymorphism is a cytosine(C) to thymine(T) transition at nucleotide position 609 exon 6 of NQO1 cDNA that encodes for a proline(P) to serine(S) substitution at position 187 in amino acid sequence of the protein (Traver et al., 1997). NQO1 C609T polymorphism is linked to the enzymatic activity to NQO1 and may affect host's susceptibility to HCC by changing the enzymatic activity. There were several studies performed to detect the association between NQO1 C609T polymorphism and risk of $\mathrm{HCC}$, but no consistent results were reported (Akkiz et al., 2010; Tan et al., 2012; Liu et al., 2013; Wang et al., 2013). We thus conducted a meta-analysis to assess the association between NQO1 C609T polymorphism and HCC risk. 


\section{Materials and Methods}

Identification and eligibility of relevant studies

We conducted a computer-assisted search with PubMed, EBSCO, the Chineses national knowledge infrastructure (CNKI), and the Wanfang databases to identify relevant published studies. We searched the databases from inception through 1 May, 2014 with the following keyword combinations: "hepatocellular carcinoma", "HCC", "NQO1 C609T", "rs1800566", and "polymorphism". Manual searches of reference lists from applicable articles were conducted to identify any studies that may have been missed by the computer-assisted search. The search was not restricted by language, but the research had to be conducted on human subjects. This study was conducted in accordance with the PRISMA guidelines (Moher et al., 2009).

Studies in this meta-analysis met the following inclusion criteria: (a) detected the relationship between the NQO1 C609T polymorphism and HCC risk; (b) case control study; (c) provided available genotype data to assess the odds ratios (ORs) and $95 \%$ confidence intervals (CIs).

\section{Data extraction}

The following data were extracted from the included studies: first author's name, year of publication, country of participants, the number of cases and controls, the number of cases and controls for each polymorphism genotype, and the $\mathrm{P}$ value for Hardy-Weinberg equilibrium (HWE). Two investigators extracted the required information from all included studies independently according to the inclusion criteria listed above. Discrepancies were resolved by discussion between two investigators.

\section{Statistical analysis}

We performed the association between NQO1 C609T polymorphism and the risk of $\mathrm{HCC}$, using allelic model ( $\mathrm{T}$ vs $\mathrm{C}$ ), additive model (TT vs CC), dominant model (TT+TC $v s \mathrm{CC}$ ), and recessive model (TT vs TC+CC). The strength of association between NQO1 C609T polymorphism and hepatocellular carcinoma risk was assessed by ORs and corresponding 95\% CIs. A chi-square based Q test was conducted to assess heterogeneity (Lau et al., 1997), and the $\mathrm{I}^{2}$ was also calculated for heterogeneity analysis (Higgins and Thompson, 2002).

To assess the quality of studies, HWE was performed in the controls using the Fisher's exact test. A P value $<0.05$ was considered statistically significant, and studies with deviation from HWE were defined as low quality studies. If the result of the $\mathrm{Q}$ test was $\mathrm{I}^{2}<50 \%$, the fixed-effects model was used; otherwise, the random-effects model was adopted. Publication bias was tested by funnel plot and Egger's linear regression test ( $p<0.05$ was considered representative of statistically significant publication bias) (Egger et al., 1997).

All analyses were performed in STATA 11.0 and RevMan 5 software. All the tests were two-sided and the significant level was 0.05 .

\section{Results}

Study selection and characteristics

We firstly identified 154 studies through the literature search. However, after reviewing of abstracts and full tests, 150 were excluded, and 4 articles were finally included into the meta-analysis (Akkiz et al., 2010; Tan et al., 2012; Liu et al., 2013; Wang et al., 2013) (Figure 1). The publication of Wang et al. (2013) presented two separate case-control studies, each study in one publication was considered separately for pooling analysis. Therefore, 4 publications including 5 studies were involved in this meta-analysis. Data were collected from 1325 patients and 1367 controls, and the main characteristics of the studies were summarized in Table 1. Among the 5 studies, 4 focused on Asians, and 1 on Caucasian.

\section{Pooled analysis}

Table 2 showed the results for the association between NQO1 C609T polymorphism and the risk of HCC. Overall, the combined results showed a significant association between NQO1 C609T polymorphism and HCC for all genetic models (allelic model: $\mathrm{OR}=1.45$, 95\% CI $=1.23-1.72, p<0.01$; additive model: $\mathrm{OR}=1.96$, $95 \% \mathrm{CI}=1.57-2.43, p<0.01$; dominant model: $\mathrm{OR}=1.62$, $95 \% \mathrm{CI}=1.38-1.91, p<0.01$; and recessive model: $\mathrm{OR}=1.53$, $95 \% \mathrm{CI}=1.26-1.84, p<0.01$ ) (Figure 2-5). In the stratified analyses by ethnicity (Asian or Caucasian), we found that the NQO1 C609T polymorphism significantly increased the risk of $\mathrm{HCC}$ in Asian populations in the all genetic models (allelic model: $\mathrm{OR}=1.50,95 \% \mathrm{CI}=1.24-1.82$, $p<0.01$; additive model: $\mathrm{OR}=2.11,95 \% \mathrm{CI}=1.48-3.01$, $p<0.01$; dominant model: $\mathrm{OR}=1.69,95 \% \mathrm{CI}=1.42-2.02$, $p<0.01$; and recessive model: $\mathrm{OR}=1.59,95 \% \mathrm{CI}=1.16$ $2.19, p<0.01$ ) (Table 2).

\section{Heterogeneity analysis}

We used Q statistic and $\mathrm{I}^{2}$ index to assess the heterogeneity between studies. As shown in Table 2, there was some heterogeneity between studies in some comparisons. We conducted a subgroup analysis, and did not identify the source of heterogeneity in the overall meta-analysis.

\section{Publication bias}

Begg's funnel plot and Egger's tests were performed

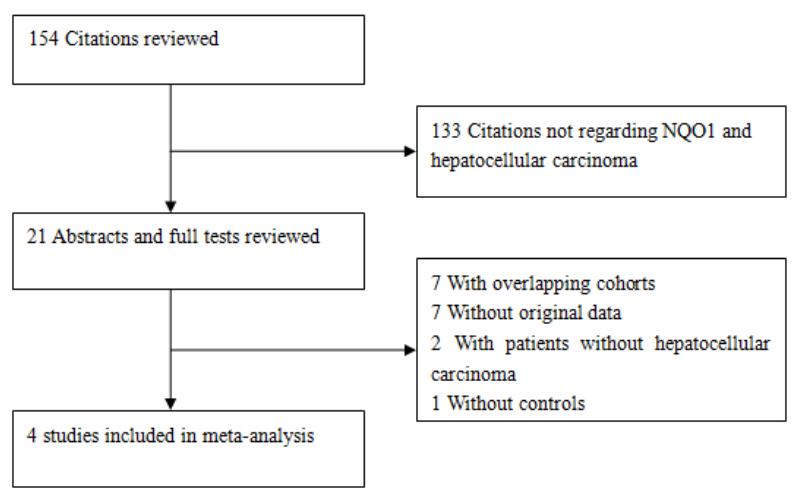

Figure 1. Flow Chat of the Study Extraction 
Table 1. Characteristics of the Studies Included in the Meta-analysis

\begin{tabular}{|c|c|c|c|c|c|c|c|c|c|c|c|c|}
\hline \multirow[t]{2}{*}{ ID } & \multirow[t]{2}{*}{ Study } & \multirow[t]{2}{*}{ Year } & \multirow[t]{2}{*}{ Country } & \multirow[t]{2}{*}{ Ethnitity } & \multirow{2}{*}{$\begin{array}{c}\text { Cases/ } \\
\text { Controls }\end{array}$} & \multicolumn{3}{|c|}{ Case } & \multicolumn{3}{|c|}{ Control } & \multirow{2}{*}{$\begin{array}{l}p \text {-HWE } \\
\text { Controls }\end{array}$} \\
\hline & & & & & & TT & $\mathrm{TC}$ & $\mathrm{CC}$ & TT & $\mathrm{TC}$ & $\mathrm{CC}$ & \\
\hline 1 & Akkiz H & 2010 & Turkish & Caucasian & $167 / 167$ & 10 & 71 & 86 & 9 & 62 & 96 & 0.81 \\
\hline 2 & Tang SK & 2012 & China & Asian & $400 / 400$ & 104 & 201 & 95 & 73 & 175 & 152 & 0.07 \\
\hline 3 & Wang WW1 & 2013 & China & Asian & $136 / 123$ & 54 & 58 & 24 & 22 & 60 & 41 & 0.1 \\
\hline 4 & Wang WW2 & 2013 & China & Asian & $146 / 151$ & 41 & 71 & 34 & 39 & 60 & 52 & 0.01 \\
\hline 5 & Liu F & 2013 & China & Asian & $476 / 526$ & 118 & 220 & 138 & 100 & 235 & 191 & 0.07 \\
\hline
\end{tabular}

Table 2. Meta-analysis of the Association between the NQO1 C609T Polymorphism and HCC

\begin{tabular}{|c|c|c|c|c|c|c|c|c|}
\hline \multirow[t]{2}{*}{ polymorphism } & \multirow[t]{2}{*}{ Studies } & \multirow[t]{2}{*}{ No. of studies } & \multicolumn{3}{|c|}{ Test of association } & \multicolumn{3}{|c|}{ Test of heterogeneity } \\
\hline & & & OR & $95 \% \mathrm{CI}$ & $p$-value & Model & $p$-value & $\mathrm{I}^{2}(\%)$ \\
\hline \multirow[t]{2}{*}{$\mathrm{T} v s \mathrm{C}$} & Overall & 5 & 1.45 & $1.23-1.72$ & $<0.01$ & $\mathrm{R}$ & 0.08 & 51 \\
\hline & Asian & 4 & 1.5 & $1.24-1.82$ & $<0.01$ & $\mathrm{R}$ & 0.07 & 57 \\
\hline \multirow[t]{2}{*}{$\mathrm{TT} v s \mathrm{CC}$} & Overall & 5 & 1.96 & $1.57-2.43$ & $<0.01$ & $\mathrm{~F}$ & 0.12 & 46 \\
\hline & Asian & 4 & 2.11 & $1.48-3.01$ & $<0.01$ & $\mathrm{R}$ & 0.09 & 53 \\
\hline \multirow[t]{2}{*}{ dominant model } & Overall & 5 & 1.62 & $1.38-1.91$ & $<0.01$ & $\mathrm{~F}$ & 0.24 & 28 \\
\hline & Asian & 4 & 1.69 & $1.42-2.02$ & $<0.01$ & $\mathrm{~F}$ & 0.25 & 27 \\
\hline \multirow[t]{2}{*}{ recessive model } & Overall & 5 & 1.53 & $1.26-1.84$ & $<0.01$ & $\mathrm{~F}$ & 0.11 & 47 \\
\hline & Asian & 4 & 1.59 & $1.16-2.19$ & $<0.01$ & $\mathrm{R}$ & 0.07 & 58 \\
\hline
\end{tabular}

Table 3. Egger's Linear Regression Test to Measure the Funnel Plot Asymmetric

\begin{tabular}{lcccc}
\hline Comparisons & Study & $\begin{array}{c}\text { Y axle intercept: } \\
\text { a(95\%CI) }\end{array}$ & t & P value \\
\hline T vs C & Overall & -15.59 & 0.41 & 0.71 \\
TT vs CC & Overall & -12.86 & 0.26 & 0.81 \\
dominant model & Overall & -12.64 & 0.65 & 0.56 \\
recessive model & Overall & -12.93 & 0.18 & 0.87 \\
\hline
\end{tabular}

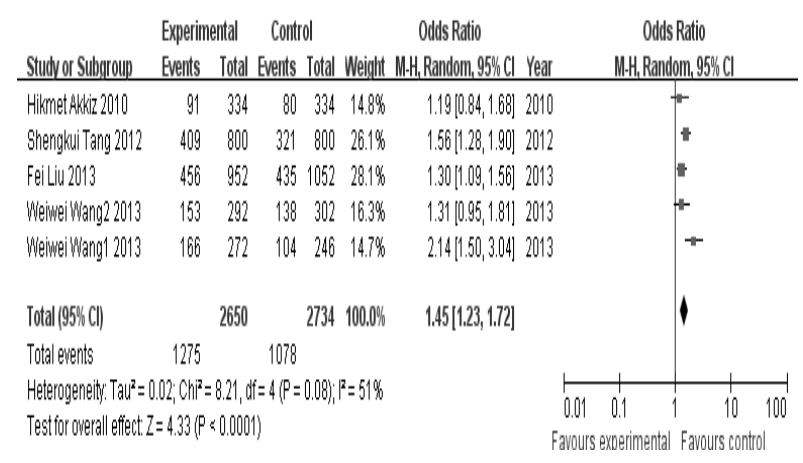

Figure 2. Forest Plots for $\mathbf{T}$ vs $\mathbf{C}$ of the Association between NQO1 C609T Polymorphism and HCC

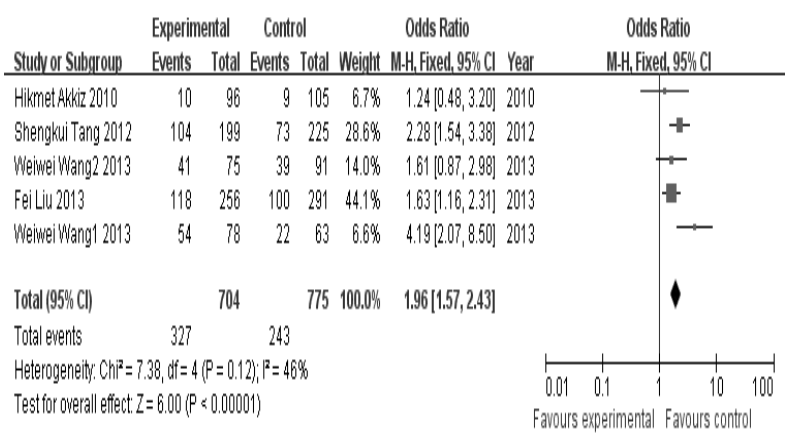

Figure 3. Forest Plots for TT vs CC of the Association between NQO1 C609T Polymorphism and HCC

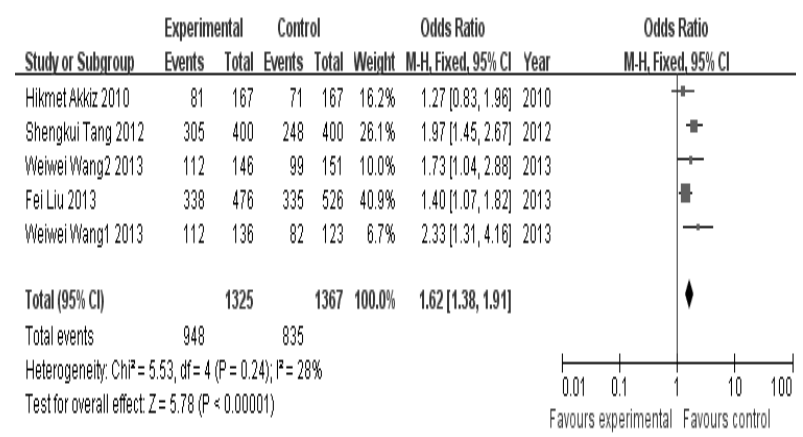

Figure 4. Forest Plots for Dominant Model (TT + TC vs CC) of the Association between NQO1 C609T Polymorphism and $\mathrm{HCC}$

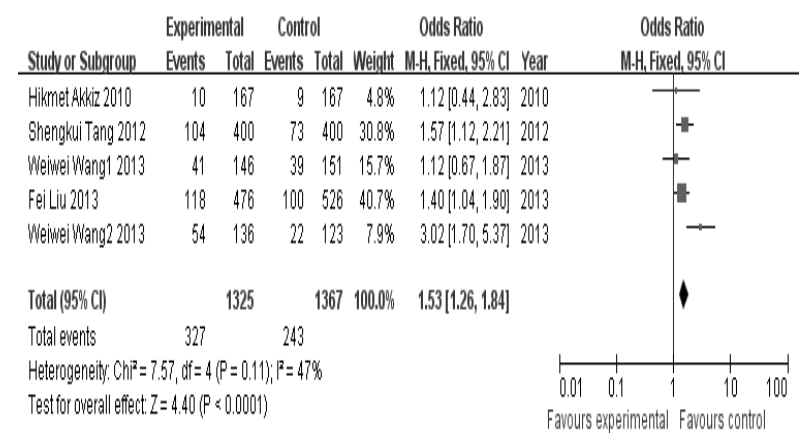

Figure 5. Forest Plots for Recessive Model (TT vs TC+CC) of the Association between NQO1 C609T Polymorphism and HCC

to assess publication bias of the literature on HCC. Figure 6 displays a funnel plot that detected the NQO1 C609T polymorphism and risk of overall HCC included in this meta-analysis in all genetic models. The shapes of the funnel plots did not show any evidence of obvious asymmetry. Also there was no statistical evidence of publication bias across studies by using Egger's linear 
Hong Zhao et al

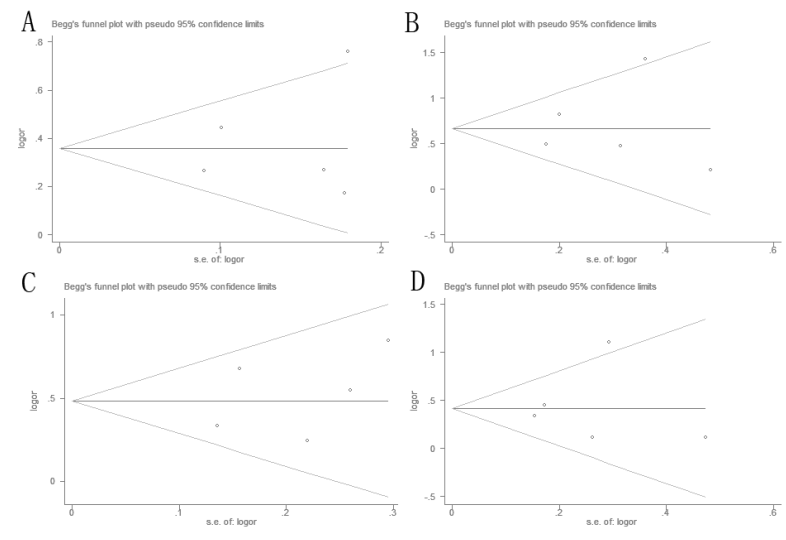

Figure 6. .Funnel Plots of All Individual Studies in the Meta-analysis of Risk of HCC with T vs C (A), TT vs CC (B), Dominant Model (C), and Recessive Model (D). No evidence of publication bias was found in any of them

regression test (allelic model: $p=0.71$; additive model: $p=0.81$; dominant model: $p=0.56$; and recessive model: $p=0.87$ ).

\section{Discussion}

The NQO1, which is generally involved in xenobiotic metabolizing, had been extensively studied on its relationship with different types of cancer, such as gastric, colorectal, cervical, prostate, and breast cancer(Hu et al., 2010; Malik et al., 2011; Yuan et al., 2011; Mandal et al., 2012; Peng et al., 2013). There were a number of studies performed to detect the association between NQO1 C609T polymorphism and HCC, but no consistent results were reported, this was largely attributed to the small samples or the relatively low statistical power of published studies. Meta-analysis is a powerful method for resolving inconsistent results with relatively large number of subjects. Therefore, this meta-analysis was conducted to provide a quantitative method for combining the different results. To the best of our knowledge, this was the most comprehensive meta-analysis assessing the genetic susceptibility of the NQO1 C609T gene polymorphism to HCC.

In the present study, we performed a meta-analysis with the 5 studies including 1325 cases and 1367 controls to explore overall effects of NQO1 C609T on HCC risk. Overall, the results of this meta-analysis from the current data suggested that there was a statistically significant association between NQO1 C609T polymorphism and an increased risk of HCC. This might be a reflection of a prominent role for NQO1 C609T variation in the etiology of HCC.

In the subgroup analysis by ethnicity, there was an obvious association between NQO1 C609T polymorphism and HCC in Asians (Table 2). In the meta-analysis, there were only 4 included studies assessing the association between NQO1 C609T polymorphism and HCC which were from Asians, and they all from Chinese. Therefore, more studies with large samples and other regions are needed to produce a more precise estimation on the effect of NQO1 C609T polymorphism on HCC.

Some limitation must be considered with interpreting the results from the meta-analysis. Firstly, although the Begg's test and Egger's test did not suggest any publication bias, selection bias could have occurred, because only studies published in English and Chinese were included in our meta-analysis. Secondly, the singlelocus-based meta-analysis precluded the possibility of gene-gene and gene-environment interactions, as well as haplotype-based effects. Thirdly, our results were based on unadjusted estimates, and therefore, they were unable to adjust them by possible confounders such as age, gender, smoking status and environment factors. Considering these potential limitations, our results should be interpreted with caution.

In summary, our pooled data show evidence for major role of NQO1 C609T polymorphism in the carcinogenesis of hepatocellular carcinoma, especially among Chinese populations.

\section{References}

Akkiz H, Bayram S, Bekar A, et al (2010). No association of NAD(P)H: quinone oxidoreductase 1 (NQO1) C609T polymorphism and risk of hepatocellular carcinoma development in Turkish subjects. Asian Pac J Cancer Prev, 11, 1051-8.

Bowen DG, Walker CM (2005). Adaptive immune responses in acute and chronic hepatitis $\mathrm{C}$ virus infection. Nature, 436, 946-52.

de Lope CR, Tremosini S, Forner A, et al (2012). Management of HCC. J Hepatol, 56, 75-87.

Dinkova-Kostova AT, Talalay P (2010). NAD(P)H:quinone acceptor oxidoreductase 1 (NQO1), a multifunctional antioxidant enzyme and exceptionally versatile cytoprotector. Arch Biochem Biophys, 501, 116-23.

Egger M, Davey Smith G, Schneider M, et al (1997). Bias in meta-analysis detected by a simple, graphical test. $B M J$, 315, 629-34.

El-Serag HB, Rudolph KL (2007). Hepatocellular carcinoma: epidemiology and molecular carcinogenesis. Gastroenterology, 132, 2557-76.

Farazi PA, DePinho RA (2006). Hepatocellular carcinoma pathogenesis: from genes to environment. Nat Rev Cancer, 6, 674-87.

Higgins JP, Thompson SG (2002). Quantifying heterogeneity in a meta-analysis. Stat Med, 21, 1539-58.

Hu X, Zhang Z, Ma D, et al (2010). TP53, MDM2, NQO1, and susceptibility to cervical cancer. Cancer Epidemiol Biomarkers Prev, 19, 755-61.

Kato N, Ji G, Wang Y, et al (2005). Large-scale search of single nucleotide polymorphisms for hepatocellular carcinoma susceptibility genes in patients with hepatitis C. Hepatology, 42, 846-53.

Kim YJ, Lee HS (2005). Single nucleotide polymorphisms associated with hepatocellular carcinoma in patients with chronic hepatitis B virus infection. Intervirology, 48, 10-5.

Lau J, Ioannidis JP, Schmid CH (1997). Quantitative synthesis in systematic reviews. Ann Intern Med, 127, 820-6.

Liu F, Luo L, Wei Y, et al (2013). A functional NQO1 609C >T polymorphism and risk of hepatocellular carcinoma in a Chinese population. Tumour Biol, 34, 47-53.

Malik MA, Zargar SA, Mittal B (2011). Role of NQO1 609C >T and NQO2-3423G $>$ A polymorphisms in susceptibility to gastric cancer in Kashmir valley. DNA Cell Biol, 30, 297303.

Mandal RK, Nissar K, Mittal RD (2012). Genetic variants in 
metabolizing genes NQO1, NQO2, MTHFR and risk of prostate cancer: a study from North India. Mol Biol Rep, 39, 11145-52.

Moher D, Liberati A, Tetzlaff J, et al (2009). Preferred reporting items for systematic reviews and meta-analyses: the PRISMA statement. Ann Intern Med, 151, 264-9.

Parkin DM (2001). Global cancer statistics in the year 2000. Lancet Oncol, 2, 533-43.

Peng XE, Jiang YY, Shi XS, et al (2013). NQO1 609C >T polymorphism interaction with tobacco smoking and alcohol drinking increases colorectal cancer risk in a Chinese population. Gene, 521, 105-10.

Siegel D, Yan C, Ross D (2012). NAD(P)H:quinone oxidoreductase 1 (NQO1) in the sensitivity and resistance to antitumor quinones. Biochem Pharmacol, 83, 1033-40.

Tan SK, Qiu XQ, Tang GF, et al (2012). Relationship between hepatocellular carcinoma and the interaction between NQO1 polymorphisms and environmental factors. Zhonghua Gan Zang Bing Za Zhi, 20, 833-7.

Traver RD, Siegel D, Beall HD, et al (1997). Characterization of a polymorphism in $\mathrm{NAD}(\mathrm{P}) \mathrm{H}$ : quinone oxidoreductase (DT-diaphorase). Br J Cancer, 75, 69-75.

Wang WW, Chen GY, Sun JJ, et al (2013). The relationship between polymorphisms of NQO1 genes and hepatocellular carcinoma in Zhengzhou and Guilin areas. Chin J Hepatobiliary Surg, 19, 836-40.

Yuan W, Xu L, Chen W, et al (2011). Evidence on the association between NQO1 Pro187Ser polymorphism and breast cancer risk in the current studies: a meta-analysis. Breast Cancer Res Treat, 125, 467-72.

Yuen MF, Hou JL, Chutaputti A, et al (2009). Hepatocellular carcinoma in the Asia pacific region. $J$ Gastroenterol Hepatol, 24, 346-53. 